\title{
THE PREVALENCE AND IMPACT OF ORO-FACIAL PAIN AMONG 12-YEAR-OLD SCHOOLCHILDREN IN AN EPIDEMIOLOGICAL SURVEY SETTING
}

N. Jaafar, I.A. Razak. The prevalence and impact of oro-facial pain among 12-year-old schoolchildren in an epidemiological survey setting. Annal Dent Univ Malaya 2002; 9: 11-16.

\section{ABSTRACT}

The objective of the study was to attempt to verify the cause of self-reported oro-facial pain among 12-yearold children, objectively via a clinical examination. This is a descriptive, cross-sectional survey using a combination of self-reported questionnaire, face-to-face interview and clinical oral examination. The children were first asked to answer a self-filled questionnaire about their oro-facial pain experience in the past 4weeks. In order to verify its cause, a clinical examination and an interview followed. Normative oral health status data was also collected. The sample was 1492 Malay schoolchildren with diverse socioeconomic background from the states of Johore, Kelantan and Sabah. The sample size for each state was calculated to give a sampling error of not more than $5 \%$. In each state, quota sampling was done to achieve a balanced distribution between gender and location. The data collected were normative status for caries, periodontal disease and traumatized teeth. Orofacial pain experience represented the subjective status for oral well-being. The cause of pain was confirmed through a clinical examination. The normative oral health status data implies a very low untreated disease and good oral health among the schoolchildren. However the subjective health status, as reflected by the prevalence of pain suggested that oro-facial pain and suffering was high (27.3\%) with about $49 \%$ "of moderate and severe" intensity. The two main causes were caries and mouth ulcers. However in about onequarter of pain cases, diagnosis cannot be confirmed in the field survey setting. More than one-half of those with pain experienced disturbed sleep and study. It was concluded that overall oral health status and well-being can be better described if normative data is complemented with subjective data such as pain prevalence. The study shows that the majority (more than $75 \%$ ) of cases of subjective pain can be objectively verified in a field epidemiology survey setting. The reliability of the subjective data can be improved by a clinical examination as compared to unverified self-report. The study also confirms that the major source of oro-facial pain among the 12 year-olds were caries and mouth ulcers.

Key words: oral epidemiology, oral health status, orofacial pain, dental pain.
Original Article

$$
\begin{aligned}
& \text { N. Jaafar }{ }^{1} \text { and I.A. Razak } \\
& \\
& { }^{1,2} \text { Professor }
\end{aligned}
$$

Department of Community Dentistry, Faculty of Dentistry, University of Malaya 50603 Kuala Lumpur, Malaysia. E-Mail: nasrudd@um.edu.m.

Fax: 6- 03- 79674532

Tel: 6- $03-79674805$

Corresponding author - Prof. Dr Nasruddin Jaafar

\section{INTRODUCTION}

Oral health status and needs is still predominantly reported in terms of normative indices such as DMFT, CPITN, prosthetic needs, fluorosis index, malocclusion index etc. However this may not really reflect the holistic view of oral well-being and quality of life. Most people do not identify whether they have good oral health by the number of DMF teeth but rather if they had experienced crippling pain or not.

The use of subjective indices to reflect the impact of oral disease on the quality of life has been suggested since the 1980's (1). However, most authors agree that there was no question of whether subjective measures should replace normative indices because neither type by itself is sufficient. Both should be considered as complementary. Together they could provide a more complete picture of the population's health status and well being (2). Many composite subjective health status indicators such as the Sickness Impact Profile (3) and Nottingham Health Profile (4) were developed and tested. Similar composite equivalents for oral health were also developed by Marcus et al, 1983 (5); Jakobsen and Hunt, 1990 (6), and Slade and Spencer, 1994 (7).

However, these composite measures although useful to researchers and academicians, are difficult to interpret by the layman and policy makers. Alternatively, data on pain prevalence and profile (including oro-facial pain) may be more relevant and easier to understand. Pain may be considered as a proxy measure of disease or can be regarded as measures in their own right (8).

To date, most of the research on oro-facial pain involved adult populations (9). Prevalence data for children was few. Bruusgaard et al (10) found that there was a strong association between pain, mental distress 
and sleep problems in schoolchildren. Among 12 yearold children in Kuwait, Vigild et al (11) reported that $28 \%$ had experienced oral health problems in the previous 12 months, mainly from pain (10\%) and discomfort (18\%). In the USA, the parents of 5-13 and 14-17 year-old children reported that 5 and $8 \%$ respectively, had pain or distress related to teeth and gums in the past 3 months (12). Parents of children aged 8-9 years-old in Canada reported in a telephone interview that $5-8 \%$ had experienced pain in the previous 4-weeks (13). In South Australia, $12 \%$ of 5year-old and $32 \%$ of 12-year-old schoolchildren reported a history of toothache, which affected their schoolwork (14). Earlier studies in the UK, reported $33,48,51$ and $49 \%$ of $5-7,8-11,12-13$ and 14-15 year-olds respectively, suffered from toothache at some time in their lives (15).

Therefore it may be considered that oro-facial pain and its impact are legitimate outcome indicators of oral health and well being. These indicators provide important information regarding the quality of life of individuals and the society by complementing the normative disease indicators. The objectives of the present study were (i) to describe the oro-facial pain profile in the previous 4-weeks, i.e. the prevalence, severity and impact, and (ii) to test the feasibility of verifying the cause of the self-reported oro-facial pain objectively through a clinical examination and casehistory interview in a field survey setting.

\section{METHOD}

The sampling frame consists of all 12 year-old Malay schoolchildren living in three Malaysian states viz. Johore, Kelantan and Sabah. The states were chosen to represent the widest range of normative dental disease experience based on available national oral health data (16). The three states were chosen to represent the best and worst normative status. The pooled data from these states would provide the best indication of the national oral health status. Two districts were chosen for each state. Schools in each district were first stratified into urban and rural. The sampling frame consists of all 12 year-old Malay schoolchildren in each state. Based on available data on the prevalence of dental disease, the sample size was calculated to represent the population of Malay school children in that state with an error not exceeding $5 \%$. In the selected schools, the children were randomly chosen until the minimum sample size was met or exceeded. Wherever possible, a roughly equal representation (50:50) of male and female samples was sought through quota sampling, rather than proportionate sampling.

Subjective data on oro-facial pain was collected in two-phases. First, the subjects were asked to fill a questionnaire about their oro-facial pain experience in the previous 4-weeks. This was immediately followed by a clinical examination and face-to-face interview to validate objectively the source of pain. Normative data for caries and periodontal status were collected using WHO basic oral health survey criteria (17).

A self-filled questionnaire was distributed to the children to fill. Subjects who claimed in the questionnaire to have suffered from oro-facial pain in the past 4-weeks were interviewed in detail about the pain experience at the clinical examination that followed. The child was asked to show the offending site. A visual and clinical examination was done to verify the cause of pain and the tentative diagnosis was recorded.

Since the method of verification is being developed for use in an epidemiological field survey setting, the use of advanced diagnostic equipment such as radiography, pulp tester and fibre-optic transillumination were excluded. The clinical examiner was only allowed to examine visually, palpate and percuss the claimed site of pain. This is intended to recreate an epidemiological field survey setting as closely as possible using only the basic examination tools viz. plane mouth mirror, curved probe (to remove debris only) and a portable light source. Based on the history and the clinical examination, the examiner was expected to give a preliminary diagnosis of the cause of pain.

The questionnaire was pre-tested on a group of students with similar characteristics and found to be adequate. For the clinical examination, $5 \%$ of the daily sample was recalled. Only one clinical examiner was used to maintain consistency. The intra-examiner consistency was acceptable as the percentage agreement in both the pre-test and actual study was at least $92 \%$. Only Malay children were involved in this study in order to eliminate ethnic variations in disease patterns and control the accuracy of understanding the questionnaire.

\section{RESULTS}

Table 1 shows the normative oral health status indicators commonly used in epidemiological surveys of 12-year-old schoolchildren. The prevalence of caries, periodontal disease and traumatic injury to anterior teeth was only $68.8 \%, 23.7 \%$ and $2.6 \%$, respectively. The mean DMFT, DT, MT and FT were $2.07,0.79,0.11$ and 1.17 , respectively, which indicated a very low untreated disease burden. The majority had healthy periodontium (76.3\%), very few bleeding gums (4.8\%) and only a small amount of calculus $(18.9 \%)$. The clinical examination indicates that the normative oral health status is good.

However the indicator of well-being was disturbing. The prevalence of oro-facial pain in the past 4-weeks was rather high (27.3\%) (Table 2). A quantitative analysis in this group with oro-facial pain $(n=408)$ indicated that the mean number of days 
Table 1. Normative oral health status indicators of 12 year-old schoolchildren

\begin{tabular}{|c|c|c|c|}
\hline Disease / Condition & Index used & Normative Data ( \pm s.d) & Overall Normative Conclusion \\
\hline \multirow[t]{5}{*}{ Dental Caries } & Prevalence $(\mathrm{N}=1492)$ & $68.8 \%$ & - Moderately high prevalence \\
\hline & Mean DMFT & $2.07(2.24)$ & - Few carious teeth \\
\hline & Mean decayed teeth (DT) & $0.79(1.59)$ & - Very few untreated decay \\
\hline & Mean missing teeth (MT) & $0.11(0.40)$ & - Very low missing teeth \\
\hline & Mean filled teeth (FT) & $1.17(1.50)$ & - Majority are treated (filled) \\
\hline \multirow[t]{4}{*}{ Periodontal Disease } & Prevalence $(\mathrm{N}=1492)$ & $23.7 \%$ & - Low prevalence in 12 yr-old \\
\hline & CPITN=0 (healthy) & $76.3 \%$ & - Majority healthy \\
\hline & CPITN=1 (bleeding) & $4.8 \%$ & - Very few bleeding gums \\
\hline & CPITN=2 (calculus) & $18.9 \%$ & - Some calculus \\
\hline \multirow[t]{8}{*}{$\begin{array}{l}\text { Traumatic fracture } \\
\text { to anterior teeth }\end{array}$} & $\begin{array}{l}\text { Prevalence }(N=1492) \\
\text { No. of teeth affected }(n=39)\end{array}$ & $2.6 \%$ & - Very low prevalence \\
\hline & 1 tooth \# & $79.5 \%$ & - Mainly only one tooth affected \\
\hline & 2 teeth \# & $20.5 \%$ & \\
\hline & 3 or more \# & none & \\
\hline & Fracture severity $(n=39)$ & & \\
\hline & Less $<1 / 3$ crown & $79.5 \%$ & - Mainly involving enamel fractures \\
\hline & $1 / 3$ to $2 / 3$ crown & $17.9 \%$ & \\
\hline & More $>2 / 3$ crown & $2.6 \%$ & \\
\hline
\end{tabular}

Table 2. Variations in overall prevalence of oro-facial pain at different recall-periods

\begin{tabular}{|c|c|c|c|c|c|c|c|}
\hline \multirow[t]{2}{*}{ Prevalence of oro-facial pain } & \multicolumn{2}{|c|}{ With pain } & \multicolumn{2}{|c|}{ No Pain } & \multicolumn{2}{|c|}{ Total } & \multirow{2}{*}{$\begin{array}{l}\text { Mean no. of pain days } \\
n=408( \pm s d)\end{array}$} \\
\hline & n & (\%) & $n$ & $(\%)$ & $N$ & $(\%)$ & \\
\hline In the past 12 months & 897 & $(60.1)$ & 595 & $(39.9)$ & 1492 & (100) & - \\
\hline In the past 4 weeks & 408 & (27.3) & 1084 & (72.3) & 1492 & $(100)$ & $2.58(2.49)$ \\
\hline On the day of clinical examination & 107 & $(7.2)$ & 1385 & $(92.8)$ & 1492 & $(100)$ & - \\
\hline
\end{tabular}

suffered in the past 4-weeks was 2.58 ( \pm SD 2.49) days. The range was between one to 21 days. However, in the majority of cases $(81 \%)$, it lasted for only 3 days or less. Table 2 also shows that the prevalence of selfreported oro-facial pain varies widely when different recall period was used. The prevalence of pain, overall, ranged from $7.2 \%$ (ie. $n=107 / 1492$ ) on the day of clinical examination to $60.1 \%$ when considered over a period of the past 12 months.

Table 3 shows the distribution of oro-facial pain by self-perceived severity in the past 4-weeks. Of those with pain, slightly more than one-half described it as "mild" and $40 \%$ as "moderate". Only about one in ten children with pain claimed that it was "severe or very severe".

Table 4 shows the reported frequency of oro-facial pain in the past 4-weeks. The majority said it occurred "sometimes or occasionally" (i.e. once or twice a day). Very few said it was "often".

Table 5 shows the cause of oro-facial pain, which was verified by the clinical examination (where possible) and based on the description gleaned from
Table 3. Distribution of oro-facial pain by perceived severity

\begin{tabular}{lrr}
\hline Perceived Severity of Oro-Facial Pain & $n$ & $(\%)$ \\
\hline Mild & 208 & $(51.0)$ \\
Moderate & 163 & $(40.0)$ \\
Severe & 35 & $(8.6)$ \\
Very severe & 2 & $(0.5)$ \\
\hline Total & 408 & $(100)$ \\
\hline
\end{tabular}

Table 4. Frequency of oro-facial pain

\begin{tabular}{lrr}
\hline Frequency of oro-facial pain & \multicolumn{1}{c}{$n$} & $(\%)$ \\
\hline Sometimes / Occasionally (1-2 X a day) & 391 & $(95.8)$ \\
Often (3X or more a day) & 17 & $(4.2)$ \\
\hline Total & 408 & $(100.0)$ \\
\hline
\end{tabular}


Table 5. Clinically verified causes of oro-facial pain

\begin{tabular}{lrr}
\hline Tentative Clinical Diagnosis & $\mathrm{n}$ & $(\%)$ \\
\hline Primary caries & 164 & $(40.2)$ \\
Oral ulcers & 96 & $(23.5)$ \\
Periodontal problems & 17 & $(4.2)$ \\
Secondary caries (recurrent) & 14 & $(3.4)$ \\
Temporo-mandibular joint pain & 10 & $(2.5)$ \\
Traumatic injury & 6 & $(1.5)$ \\
Abrasion cavity & 1 & $(0.2)$ \\
Others (undiagnosable causes) & 100 & $(24.5)$ \\
\hline Total & 408 & $(100.0)$ \\
\hline
\end{tabular}

Table 6. Physical and social impact of oro-facial pain (Base $\mathrm{N}=408$ )

\begin{tabular}{lrr}
\hline Type of impacts * & $\mathrm{n}$ & $(\%)$ \\
\hline Avoided certain foods & 201 & $(49.3)$ \\
Disturbed sleep & 86 & $(21.1)$ \\
Affected study & 76 & $(18.6)$ \\
Consulted a doctor or dentist & 85 & $(20.8)$ \\
Tried self-medication (painkiller pills) & 180 & $(44.1)$ \\
Tried traditional remedies & 11 & $(2.7)$ \\
Stayed at home & 65 & $(15.9)$ \\
Avoided friends & 27 & $(6.6)$ \\
\hline
\end{tabular}

*A subject may report one or more impacts.

the case history. On clinical examination, dental caries, both primary (40.2\%) and recurrent $(3.4 \%)$, accounted for the most common cause of oro-facial pain among schoolchildren. This was followed by oral ulcers (aphthous ulcers) based on the case history or clinical examination. Other causes are not very significant in terms of frequency. However, it is important to note that a relatively large number of cases could not be diagnosed $(24.5 \%)$ in the field-survey setting.

Table 6 shows selected physical and social impacts of oro-facial pain reported by the children in the past 4-weeks. About one-in-two children avoided foods or drinks which aggravated the pain and took over-thecounter pain killers for pain relief. Oro-facial pain affected their sleep, study and resulted in a consultation with a health professional in about onein-five children. However, the social impact such as avoided friends, stayed at home or tried traditional remedies were very few among children.

\section{DISCUSSION}

Critics of the use of subjective measures of health (including pain) are often skeptical of the validity and accuracy of these self-reported data for several reasons. First, self-reported measures such as pain experienced is said to be not very reliable because no studies have attempted to objectively verify the source of the pain through any clinical examination. A clinical examination will reveal a visible source. It has to be assumed that the subject is telling the truth. However there is the possibility that some patients might exaggerate their pain experience or the pain may be psychogenic. Second, the literature on oro-facial pain relied on the accuracy of patients' recall-memory, the duration of which varies too much. It may be expected that the longer the recall period the less reliable it would be.

Third, the method of data collection used in the literature varied from phone interviews, self-filled questionnaire and direct face-to-face interview. But none has attempted to verify the cause of pain objectively through clinical examination in a field epidemiological survey setting. The reliability of the prevalence data is again questionable. Thus there is a need to test the feasibility of a method to verify objectively the claimed sources of pain.

Fourth, there is no agreement on a suitable timeframe for reporting of past pain prevalence. The window period reported in the literature ranged from "over a life-time", "one-year", "3-months" or "in the past-4-weeks". Hence the prevalence data reported were not comparable between populations and difficult to interpret. This is not good for the advancement of global data collection on oro-facial pain. There is a need for some form of standard data collection method in order to be useful as an epidemiological tool and to set national or global goals for oral health outcomes.

Fifth, although some studies reported on the severity and impact of pain, there was no attempt to establish the cause of pain. For example, Shepherd et al assessed the prevalence, severity and impact of dental pain in the previous 4 weeks through interviews of 8 year-olds in England. They found that although $47.5 \%$ claimed to have ever suffered from toothache, only $7.6 \%$ had it in the past-4-weeks (18). They also recommended that future work should attempt to separate the prevalence of dental pain caused by physiological, from those caused by avoidable pathological factors. This was based on the premise that some types of oral pain are unavoidable and not preventable. This type of information is important for planning oral health promotion strategies by separating the "preventable" from the "inevitable". Therefore this implies that if a meaningful and comparable global database of oro-facial pain is to be built up by a central coordinating body such as the WHO Global Oral Health Data Bank, there should be some form of standardization of the method of data collection. Such global databanks are already available for most oral disease.

Based on the literature review, the present study adopted and tested the following parameters: (i) the standard window period time-frame is "the past-4- 
weeks", and (ii) the cause of self-reported pain should be verified as far as possible with a clinical examination. The smooth conduct of the study showed that the proposed method was feasible and practicable.

"Four weeks" was chosen because the present study shows that different recall periods produced different prevalence figures. The standard "past-4weeks" period was proposed because it was reasonable, reliable and verifiable. Most people would not remember events in detail very clearly more than one month ago, especially in children. If a "one-year period" was used, about $60 \%$ said they experienced pain in the present study. However there was no way of verifying the cause because the signs and symptoms of disease would have disappeared or lesions healed.

The present study proved that the 4-week period is adequate and reliable since over $75 \%$ of pain cases could be confirmed objectively by observing the clinical signs. The rest could not be diagnosed for the following reasons. First, advanced diagnostic tools such as radiographs, transillumination and pulp testing were not used. Second, some soft tissue lesions which are transient in nature (for eg. apthous ulcers) may have healed leaving no scars or bruises although that was partly overcome by the case history interview. Third, the pain may be psychogenic or idiopathic in nature or some subjects may not be truthful in their reporting. However it has to be accepted that there is no other way of checking the latter.

The prevalence data cannot be compared to other international studies, as the evidence among 12 yearold school children was scarce. The present study could provide an important baseline for future studies to compare. The present study revealed that just over one-quarter of all children in the sample $(n=408$, $27.3 \%$ ) experienced oro-facial pain in the past 4weeks. Of these, $107(7.2 \%)$ still had the pain on the day of examination. This gives the policy makers a clearer perspective of the burden of pain and suffering caused by oral diseases. For example, if this proportion was extrapolated, assuming that $7 \%$ of 12 year-old schoolchildren is suffering from oro-facial pain at any one time (point prevalence), this may be multiplied by the number of children in this age-group. The actual number of sufferers from preventable pain may be considerable relative to other general health problems. This gives a more holistic picture of overall health status from the public health point of view, as compared to relying on normative clinical data alone.

Based on the present profile of oro-facial pain, the rosy normative health status data may hide the fact that the oral health-related quality of life may not be that good after all. More can be done. Fortunately the majority of pain-cases (about $91 \%$ ) are only described as "mild or moderate", occurring only "occasionally" and lasting for at most, two or three days. However, the impact at the personal level is disturbing because one-in-two children avoided foods and drinks which will aggravate the pain and just as many said they resorted to pain killers. About one-in-five had their sleep and study affected by the pain. This supports the view that oral diseases although not life threatening may impose a great burden on the population's wellbeing (9). However it appears that in most cases of oro-facial pain, it did not affect the children's socializing activities such as playing with friends.

The present study concluded that in order to reduce orally-related pain, suffering and its effect on the quality of life among 12-year-old schoolchildren, the oral healthcare services in Malaysia should further emphasize on the prevention of dental caries since this has been identified as the main contributor of preventable oro-facial pain. As for mouth ulcers, which is the second most important contributor, palliative services should be made available in the school dental service since they are neither preventable nor easily treated. It is also suggested that the proposed guidelines to standardize international research on the epidemiology of oro-facial pain be promoted and used internationally.

\section{ACKNOWLEDGEMENTS}

We wish to acknowledge the assistance of the Oral Health Division Ministry of Health Malaysia and schools in the Ministry of Education in Kelantan, Johore and Sabah involved in the survey. The research was funded by Research Grants (F107/94, F227/95 and F249/96) received from the University of Malaya.

\section{REFERENCES}

1. Reisine, S. Theoretical considerations in formulating sociodental indicators. Soc Sci and Med 1981; 15A, 745-50.

2. Andrews, F.M. Social indicators and health for all. Soc Sci and Med 1981;15C: 219-23.

3. Bergner, M. Bobbitt, R.A., Kressel, S., Pollard, W.E., Gilson, B.S. and Morris, J.R. The sickness impact profile: conceptual formulation and methodology for the development of a health status measure. Int J Health Serv 1976; 6: 393-415.

4. Hunt, S.M., McKenna S.P., McEwen, J, Williams, J. and Papp, E. The Nottingham Health Profile: subjective health status and medical consultations. Soc Sci and Med 1981; 15A: 2219.

5. Marcus, M., Koch, A.L. and Gershen, J.A. A proposed index of oral health status: a practical application. J Amer Dent Assoc 1983; 107: 729733. 
6. Jakobsen, J.R. and Hunt, R.J. Validation of oral status indicators. Community Dent Health 1990; 7: $279-84$

7. Slade, G.D. and Spencer, A.J. Development and evaluation of the Oral Health Impact Profile. Community Dent Health 1994; 11: 3-11.

8. Locker, D. Measuring oral health: a conceptual framework. Community Dent Health 1988; 5, 318.

9. Reisine, S. and Locker, D. Social, psychological and economic impacts of oral conditions and treatment. In Cohen, L.K and Gift, H.C. (editors). Disease Prevention and Oral Health Promotion. Copenhagen: FDI / Munksgaard, 1995; pp 33-71.

10. Bruusgaard, D., Smedbraten, B.K. and Natvig, B. Bodily pain, sleep problems and mental distress in schoolchildren. Acta Paediatrica 2000; 89: 597 600 .

11. Vigild M, Petersen P.E., Hadi R. Oral health behaviour of 12 year old children in Kuwait. International J Paediatric Dent 1999; 9: 23-9.

12. Bailit, H.L. The prevalence of dental pain and anxiety: their relationship to quality of life. New York State Dent J 1987; 53: 27-30.
13. Woodward G.L., Leake, J.L. and Main, P.A. Oral health and family characteristics of children attending private or public dental clinics. Community Dent Oral Epidemiol 1996; 24: 2539.

14. Slade, G.D., Spencer, A.J., Davies, M.J. and Burrow, D. Intraoral distribution and impact of caries experience among South Australian schoolchildren. Australian Dent J 1996; 41: 343350.

15. Todd, J.E. Children's dental health in England and Wales 1973. London: HMSO 1975.

16. HMIS (Health Management Information System) Annual Report 1994 Dental Sub-system. Kuala Lumpur: Health System and Documentation Unit, Ministry of Health Malaysia. 1995.

17. WHO (World Health Organization). Oral Health Surveys Basic Methods $3^{\text {rd }}$. Edition. Geneva: World Health Organization 1987.

18. Shepherd, M.A., Nadanovsky, P. and Sheiham, A. The prevalence and impact of dental pain in 8year-old schoolchildren in Harrow, England. Brit Dent J 1999: 187: 38-41. 